\title{
Coherent light from E-field induced quantum coupling of exciton states in superlattice- like quantum wells
}

Lyssenko, V. G.; Østergaard, John Erland; Hvam, Jørn Märcher; Löser, Falk; Leo, Karl; Köhler, K.

Published in:

Proceedings of QELS'99

Link to article, DOI:

10.1109/QELS.1999.807096

Publication date:

1999

Document Version

Publisher's PDF, also known as Version of record

Link back to DTU Orbit

Citation (APA):

Lyssenko, V. G., Østergaard, J. E., Hvam, J. M., Löser, F., Leo, K., \& Köhler, K. (1999). Coherent light from Efield induced quantum coupling of exciton states in superlattice-like quantum wells. In Proceedings of QELS'99 (pp. 12-13). IEEE. https://doi.org/10.1109/QELS.1999.807096

\section{General rights}

Copyright and moral rights for the publications made accessible in the public portal are retained by the authors and/or other copyright owners and it is a condition of accessing publications that users recognise and abide by the legal requirements associated with these rights.

- Users may download and print one copy of any publication from the public portal for the purpose of private study or research.

- You may not further distribute the material or use it for any profit-making activity or commercial gain

- You may freely distribute the URL identifying the publication in the public portal 
neous applied stress to create a potential energy minimum for the excitons via the deformation potential. This method bears some similarity to an earlier method used to create a harmonic potential minimum for excitons in bulk semiconductors (e.g. Ref. 2), but in our case, the primary effect of the applied force is not to create shear stress, but to create a hydrostatic expansion induced by a slight bending of the sample. Since the hydrostatic deformation potential of the conduction band in GaAs is large and negative, a hydrostatic expansion leads to a large reduction of the conduction electron energy. This implies that this method can be used not only for excitons, but also for free conduction-band electrons. This could lead to new experiments on the twodimensional electron gas in a harmonic potential, using applied stress (which can be varied continuously in our experiments) as a new type of gate to control the depth of the trap.

We have used both of these methods to create in-plane harmonic potential minima for indirect excitons in GaAs coupled quantum wells consisting of two wells each $60-100 \AA$ wide, separated by a $40 \AA \mathrm{Al}_{x} \mathrm{Ga}_{1} .{ }_{x}$ As barrier. The resulting traps have a depth of up to 10 $\mathrm{meV}$ (allowing confinement of the excitons at temperatures up to $100 \mathrm{~K}$ ) and spatial dimension of the order of $200 \mathrm{mum}$. Since the excitons move in response to an electric field, we can assign them a mobility, which we measure optically as $1000 \mathrm{~cm}^{2} / \mathrm{V}-\mathrm{s}$ at $100 \mathrm{~K}$. At high carrier density, we also optically measure diffusion constants of the excitons of up to 1000 $\mathrm{cm}^{2} / \mathrm{s}$.

The ability to produce an in-plane harmonic potential for the exciton gas represents an important step toward the realization of theoretical proposals (e.g. Ref. 3) for Bose condensation of two-dimensional excitons in a confining potential.

This work has been supported by the $\mathrm{Na}$ tional Science Foundation and by the Research Corporation, which has supported D.S. as a Cottrell Scholar.

${ }^{*}$ Max-Planck Institut für Festkörperforschung, Heisenbergstr. 1, 70506 Stuttgart, Germany

1. M. Hagn, A. Zrenner, G. Böhm, and G. Weimann, Appl. Phys. Lett. 67, 232 (1995).

2. P.L. Gourley and J.P. Wolfe, Phys. Rev, B 24, 5970 (1981).

3. X.J. Zhu, P.B. Littlewood, and T.M. Rice, Phys. Rev. Lett. 74, 1633 (1995).

Coherent light from E-field induced quantum coupling of exciton states in superlattice-like quantum wells

V.G. Lyssenko, J. Erland, J.M. Hvam, F. Löser, ${ }^{*}$ K. Leo, ${ }^{\star}$ and K. Köhler, ${ }^{* *}$ Mikroelektronik Centret, The Technical University of Denmark, Building 345E, DK2800 Lyngby, Denmark; E-mail: jeo@mic.dtu.dk

Coherences and electronic couplings at the quantum level in semiconductor nanostruc-

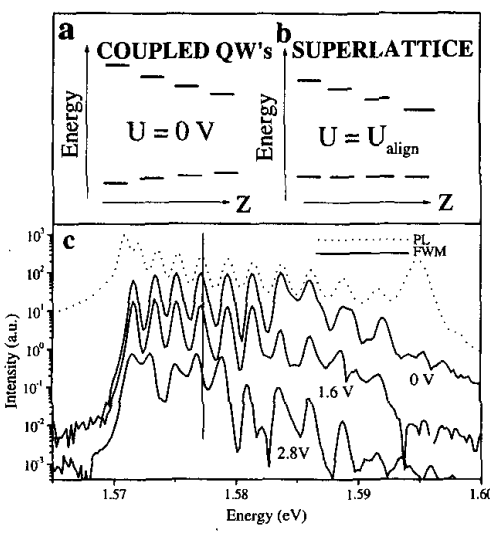

QMC3 Fig. 1. Sketch of the electron and hole levels in the GaAs multiple quantum well sample without (a) and with (b) an external bias field. (c) The photoluminescence spectrum (dotted) excited by a HeNe laser showing the 13 first lines of the single quantum wells at $5 \mathrm{~K}$. Also shown are the four-wave mixing spectra at three different bias voltages. The vertical line is a guide to the eye to shown the shift of the lines as a function of bias voltage.

tures are currently investigated intensely because of fundamentally new properties that may become important for new optoelectronic devices. Examples include observations of $\mathrm{THz}$ radiation from double quantum wells, ${ }^{\prime}$ coherent control of single quantum dot states, ${ }^{2}$ Bloch oscillations in superlattices, ${ }^{3}$ and molecular-like spectra from coupled quantum dots. ${ }^{4}$ In this work we focus on the ability to control the electronic coupling in coupled quantum wells with external E-fields leading to a strong modification of the coherent light emission, in particular at a bias where a superlattice-like miniband is formed. More specifically, we investigate a MBE-grown GaAs sample with a sequence of 15 single quantum wells having a successive increase of 1 monolayer in width ranging from $62 \AA$ to $102 \AA$ and with AlGaAs barriers of $17 \AA$. The electron and hole states without bias can therefore be sketched as in Figure 1(a) and indeed almost spectrally equidistant photoluminescence lines are observed as seen in Figure 1(c). Because of excitation well above the bandgap, creating free carriers effectively screening an externally applied field, we did not observe any spectral change as the bias field was changed. ${ }^{5}$ In resonant four-wave mixing experiments, however, the spectra in Figure 1(c) clearly show consistent shifts of the individual lines as the bias field is increased. Focussing on the spectrally lowest line, we can observe very fast oscillations in the coherent emission around a bias voltage of $1.6 \mathrm{~V}$, see Figure 2(a). The inset shows the FWM spectrum indicating that the broad fs' laser excites at least 8 lines significantly. The beat period of $380 \mathrm{fs}$ can not be explained by interference between any two individual lines as the period corresponds to a bandwidth of $10.9 \mathrm{meV}$ much more than the line splitting. Rather, this shows that around this voltage the hole levels have been aligned as sketched in Figure 1(b) to induce strong electronic coupling between the quantum wells

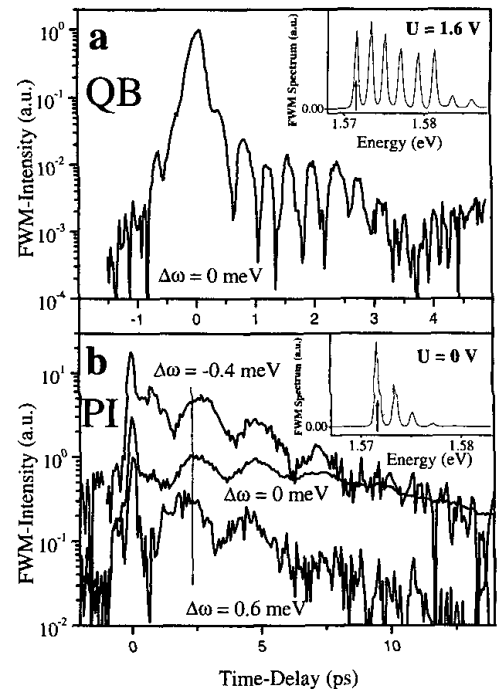

QMC3 Fig. 2. Four-wave mixing traces as a function of time-delay between the two incident pulses at and around the lowest line indicated by the arrow in the insets of the four-wave mixing spectrum. (a) At a bias voltage of $1.6 \mathrm{~V}$ and zero detuning $(\Delta \omega=0 \mathrm{meV})$ showing fast beats that can be attributed to quantum beats (QB). (b) At zero bias voltage and for three different detunings showing the phase change of the beats, indicated by the vertical line, as a result of polarization interference (PI).

resulting in the fast quantum beat. The measured beat bandwidth corresponds to having aligned the first six levels so they coherently contribute to the beats observed in Figure 2(a). In contrast to this we find much longer beat periods of $2.3 \mathrm{ps,} \mathrm{corresponding} \mathrm{to} \mathrm{the} \mathrm{level}$ splitting of $1.8 \mathrm{meV}$, without a bias field as shown in Figure 2(b), where the laser has been detuned slightly to excite only the lowest levels. Moreover, the phase change for small detunings around the lowest level ${ }^{6}$ shows that these beats are mere polarization beats in the external detector and not due to any electronic coupling between the four excited levels. Thus we have shown that indeed we can externally control the degree of electronic coupling in superlattice-like quantum wells. Further studies are in progress to clarify in detail how externally applied E-fields can modify the electronic states in these types of nanostructures relevant for applications in optoelectronic devices and in particular when minibands are formed.

*Institut fur Angewandte Photophysik, TU, 01062 Dresden, Germany

${ }^{*}$ Fraunhofer-Institut für Angewandte Festkorperphysik, 79108 Freiburg, Germany

1. K. Leo, J. Shah, E.O. Göbel, T.C. Damen, S. Schmitt-Rink, W. Schäfer, K. Köhler, Phys. Rev. Lett. 66, 201 (1991).

2. N.H. Bonadeo J. Erland, D. Gammon, D.S. Katzer, D. Park and D.G. Steel, Science. Nov. 1998.

3. V.G. Lyssenko G. Valusis, F. Löser, T. Hasche, K. Leo, M.M. Dignam, and K. Köhler, Phys. Rev. Lett. 79, 301 (1997).

4. W. Wegscheider, G. Schedelbeck, G. Abst- 
reiter, M. Rother, and M. Bichler, Phys. Rev. Lett. 79, 1917 (1997).

5. S. Farad, E. Fortin, and J.L. Merz, Phys. Rev. B 48, 11062 (1993).

6. V.G. Lyssenko, J. Erland, I. Balslev, K.-H. Pantke, B.S. Razbirin, and J.M. Hvam, Phys. Rev. B 48, R5720 (1993).

\section{QMC4}

8:45 am

Rabi oscillations and Raman coherences in semiconductor quantum wells

R. Binder, Optical Sciences Center, University of Arizona, Tucson, Arizona 85721 USA

Optical Rabi oscillations are strictly defined in terms of two-level systems (atoms or molecules) that are exposed to a stationary coherent light field in or close to resonance. In these systems the population oscillates harmonically between the lower and the upper electronic level. The observation of excitonic Rabi oscillations (i.e., the semiconductor analog to the case of atomic 2-level systems) have been achieved only recently ${ }^{1,2}$ (see also the invited talk by Schülzgen $e t$ al. at this conference).

In contrast to atomic two-level systems and their semiconductor 2-band counterpart, the study of 3-level systems and their semiconductor 3-band counterpart is of fundamental importance in the area of light-induced non-radiative quantum coherences (Raman coherences), which cannot occur in 2-level systems.

In this contribution, we investigate theoretically the relation between generalized Rabi oscillations involving optical transitions in threeband systems and Raman coherences. The theoretical basis can be found in Ref. 3. The system under consideration is a conventional GaAs semiconductor quantum well in which only the lowest subband of the conduction, the heavyhole (hh) and the light-hole (lh) bands need to be taken into account. We consider two simulateneous, strong sub-picosecond pulses (770 fs) of opposite circular polarization, spectrally centered at the hh and the lh-exciton, respectively (see Figure 1). In this configuration we create both, the intervalence-band Raman coherence and excitonic optical Rabi oscillations. In order to balance the lower lh-oscillator strength as compared to the hh-exciton, the amplitude of the pulse centered at the lh-exciton has been chosen to be three times larger than that of the other pulse. Figure 2(a) documents the 3-band Rabi

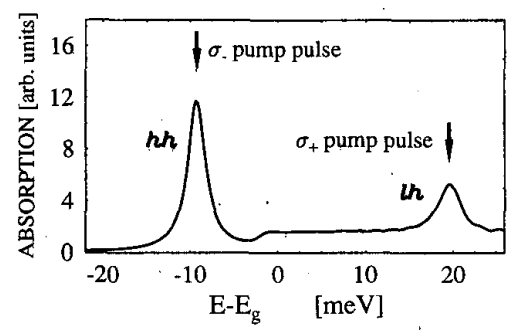

QMC4 Fig. 1. Schematic of the optical configuration. Shown is the linear absorption spectrum with heavy-hole (hh) and light-hole (lh) excitons, and the center frequencies of the two simultaneous pump pulses. $\mathrm{E}_{g}$ denotes the hhbandgap.
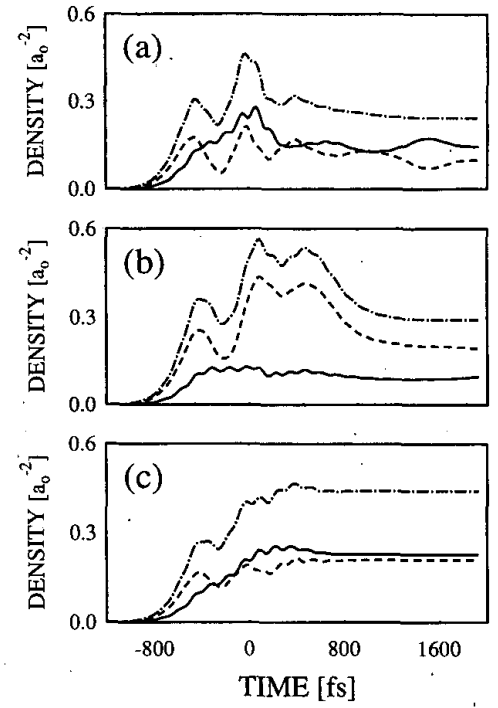

QMC4 Fig. 2. Calculated densities vs time for the spin $1 / 2$ conduction band (dash-dotted lines), and the heavy-hole (solid lines) and lighthole (dashed lines) bands that are dipole coupled to this conduction band $\left(a_{o}=135 \AA \AA\right.$ ). (a) Without dephasing, including intervalence band coherences, (b) without both, dephasing and intervalence band coherences, $(c)$ with dephasing $\left(T_{2}\right.$ $=526 \mathrm{fs}$ ) and intervalence band coherences.

oscillations, occuring for the electron, the hh and the lh density. In Figure 2(a), no incoherent processes are taken into account so that the ideal quantum coherence can be analyzed properly. The fast and somehow irregularly looking oscillations on top of the basic and slow Rabi oscillations are hh-lh quantum beats. In Figure 2(b) the results without Raman coherence show an almost complete suppression of the hh-Rabi oscillations for this choice of light-pulse amplitudes. Figure 2(c) shows the effect of dephasing, which, as usual, reduces significantly the contrast in the Rabi oscillations.

1. S.T. Cundiff, A. Knorr, J. Feldmann, S.W Koch, E.O. Göbel; and H. Nickle, Phys. Rev. Lett. 73, 1178 (1994).

2. A. Schülzgen, R. Binder, M. Donavan, $M$ Lindberg, K. Wundke, H. Gibbs, G. Kithrova, and N. Peyghambarian, (1998) to be submitted.

3. R. Binder and M. Lindberg, Phys. Rev. Lett. 81, 1477 (1998).

\section{QMC5 (Invited)}

9:00 am

Laser-induced Rabi oscillations in semiconductors

A. Schülzgen, R. Binder, M.E. Donovan, M. Lindberg, ${ }^{*}$ K. Wundke, H.M. Gibbs, G. Khitrova, N. Peyghambarian, University of Arizona, Optical Sciences Center, Tucson, Arizona 85721 USA; E-mail: axel@u.arizona.edu

The microscopic understanding of coherent light-matter interactions is an important goal of solid-state physics which can be exploited in ultrafast optoelectronics. One of the most fun-
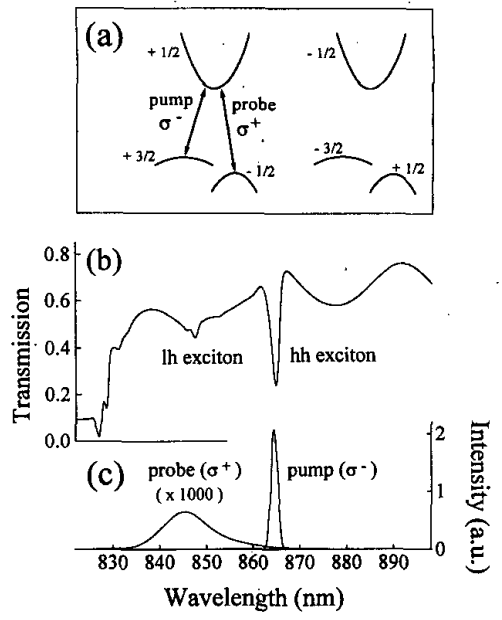

QMC5 Fig. 1. (a) Pump-probe configuration for a 6-band semiconductor quantum well. (b) Linear transmission spectrum of the $\mathrm{In}_{0.1} \mathrm{Ga}_{0.9} \mathrm{As} / \mathrm{GaAs}$ multiple quantum well at $5 \mathrm{~K}$. (c) Spectra of pump and probe pulses generated by two optical parametric amplifiers.

damental coherent effects is that of Rabi oscillations, whereby a strong resonant field induces temporal oscillations of the electron density between ground and excited state. ${ }^{1}$ This effect has been studied extensively in atomic and molecular two-level systems. Although, semiconductors differ from ideal twolevel systems due to mutual interactions of the extended electronic excitations, modified Rabi oscillations have been predicted theoretically to occur also in semiconductors. ${ }^{2}$ However, the short relevant time scales make experimental observation difficult. In previous studies the observations were limited to one or two density maxima due to ultrafast scattering times below $100 \mathrm{fs}^{3,4}$

We apply a two-color pump-probe scheme (see Figure 1) that enables the observation of several cycles of clearly resolved excitonic Rabi oscillations in a semiconductor quantum well. A $770 \mathrm{fs} \sigma^{-}$circularly polarized pump pulse with a narrow spectrum excites resonantly heavy hole (hh) excitons consisting of $\mathbf{m}_{\mathrm{j}}=$ $+3 / 2$ holes and $m_{j}=+1 / 2$ electrons. The electron population and its dynamics are probed using a $150 \mathrm{fs} \sigma^{+}$pulse with a center frequency at the light hole (lh) exciton transition. The linear transmission spectrum of the $\mathrm{In}_{0.1} \mathrm{Ga}_{0.9} \mathrm{As} / \mathrm{GaAs}$ sample and the pulse spectra are shown in Figure 1(b) and (c), respectively.

Figure 2(a) shows the differential transmission signal (DTS) of the probe pulse at the position of the lh exciton resonance. While the pump pulse drives the population oscillations the shorter probe pulse is timegating the transmission changes. We can clearly resolve a sequence of eight Rabi oscillations. In contrast to previous work, where 100 fs pulses were used, ${ }^{3,4}$ our long pump pulses have large (several $\pi$ ) areas at relatively small field intensities. Correspondingly, the induced carrier densities are quite low resulting in weak excitation-induced dephasing, and, accordingly, long time win- 